ABSTRACT: Introduction: Pixelated vision or visual snow has been associated with schizophrenia (Silverstein 2011). The impact of viewing a $3 \mathrm{D}$ motion picture on such a visualphenomenon has not heretofore been described.

METHOD: Case Study: A 28 year old right handed single male three years prior to presentation noticed that all his vision was pixelated. The pixelated vision is panoramic, involving the entire visual field. The pixels are characterized by 10,000 flat white and gray dots measuring $1 \mathrm{~mm} x$ $1 \mathrm{~mm}$. No changes in color, shape, or size were noted in high and low intensity light. White, dark, gray, or multicolored backgrounds had no effect on his vision. The visual distortions are not impacted by head movements, emotions, degree of tiredness, driving, or his hedonic perception of the object being visualized. The pixels were noted to disappear upon closure of both eyes but persisted during monocular vision with either eye. These visual hallucinations were sporadic during the first year and became continuous over the following two years. Two weeks after onset ofpixelated vision he developed auditory hallucinations and hyperacusis. These increased in intensity and frequency to 500-600 times per day. $\mathrm{He}$ denied palinopsia, migraines, tinnitus, and photophobia. These hallucinations persisted despite treatment with aripiprazole, paliperidone, lurasidone, olanzapine, clozapine, ziprasidone, benztropine, bupropion, lamotrigine, modafinil, trazodone, atomoxetine, and amphetamine.

RESULTS: Abnormalities in Examination: Hypoverbal, blunted affect, impaired concentration, preoccupied with racing thoughts. Admitted to actively having auditory and visualhallucinations, without suicidal or homicidal ideations. Memory testing: Able to recall 2 out of 4 objects in 3 minutes and 3 out of 4 with reinforcement. Similarities interpreted concretely. Visual Acuity: 20/20 OU. Retinal examination: Normal. Intraocular Pressure: $19 \mathrm{~mm} \mathrm{OD,} 20 \mathrm{~mm}$ OS (normal). Automotive Perimetry Testing: Normal. Cover/Uncover: Normal. Near Convergence: 3 inches (normal). Lens or filtered prism have no effect on visual snow. MRI of his brain, EEG, BAER, liver function tests, CBC, vitamin B12, folate, and thyroid function tests were normal. MRA: mild hypoplasia of distal right vertebral artery.

DISCUSSION: Visual snow has been anecdotally described as static, continuous, and independent of the specific visual environment (McKendrick, 2017). However, thepersistence of visual snow in the presence of $3 \mathrm{D}$ movies has never been reported. The visual snow paralleled auditory hallucinations and hyperacusis in frequency and intensity, which suggests there may be generalized hyperexcitability of the brain inducing both auditory and visual hallucinations. Agents that reduce cortical hyperexcitability (i.e., anticonvulsants, anxiolytics) may have efficacy. Treatment with these agents has been described (Ghannam, 2017), warrants further investigation.

FUNDING ACKNOWLEDGEMENTS: No funding.

149

\section{Deutetrabenazine for the Treatment of Tardive Dyskinesia: Results From an Open-Label, Long- Term Study}

Karen E. Anderson, $\mathrm{MD}^{\prime}$; Mat D. Davis, PhD ${ }^{2}$; Stewart A. Factor, $D O^{3}$; Robert A. Hauser, $M D, M B A^{4}$; L. Fredrik Jarskog, $M D^{5}$; Joohi Jimenez-Shahed, $M D^{6} ;$ Rajeev Kumar, MD; FRCPC ${ }^{7}$; Stanislaw Ochudlo, MD, PhD ${ }^{8}$; William G. Ondo, $M D^{9}$; and Hubert H. Fernandez, $M D^{10}$

${ }^{1}$ Georgetown University, Washington, District of Columbia, USA

${ }^{2}$ Teva Pharmaceutical Industries, Frazer, Pennsylvania, USA

${ }^{3}$ Emory University, Atlanta, Georgia, USA

${ }^{4}$ University of South Florida Parkinson's Disease and Movement Disorders Center, Tampa, Florida, USA

${ }^{5}$ University of North Carolina School of Medicine, Chapel Hill, North Carolina, USA

${ }^{6}$ Baylor College of Medicine, Houston, Texas, USA

${ }^{7}$ Rocky Mountain Movement Disorders Center, Englewood, Colorado, USA

${ }^{8}$ University Clinical Center of Silesian Medical University, Katowice, Poland

${ }^{9}$ Methodist Neurological Institute, Houston, Texas, USA

${ }^{10}$ Cleveland Clinic, Cleveland, Ohio, USA

ABSTRACT: Introduction: Tardive dyskinesia (TD) is an involuntary movement disorder resulting from exposure to dopamine-receptor antagonists. In the 12-week ARMTD and AIM-TD studies, deutetrabenazine demonstrated significant improvements in Abnormal Involuntary Movement Scale (AIMS) scores at Week 12 compared with placebo, and was generally well tolerated.

OBJECTIVE: To evaluate the efficacy and safety of longterm deutetrabenazine therapy in patients with TD.

METHODS: Patients with TD who completed the ARM-TD or AIM-TD studies were eligible to enter this openlabel, single-arm, long-term safety study after they completed the 1-week washout period and final evaluation in the blinded portion of the trial. Efficacy endpoints included the change in AIMS score from baseline, and treatment success (defined as "much improved" or "very much improved") on the Clinical Global Impression of Change (CGIC) and Patient Global Impression of Change (PGIC). This analysis reports results up to Week 54. 
RESULTS: 304 patients enrolled in the extension study. At Week 54, the mean (standard error) change in AIMS score was -5.1 (0.52). After 6 weeks of deutetrabenazine treatment, the proportion of patients who achieved treatment success was $58 \%$ per the CGIC and $53 \%$ per the PGIC, and by Week 54 was $72 \%$ per the CGIC and $59 \%$ per the PGIC, thus demonstrating maintenance or enhancement of benefit over time. Deutetrabenazine was well tolerated for up to 54 weeks, and compared with the ARM-TD and AIM-TD studies, no new safety signals were detected.

CONCLUSIONS: 54 weeks of deutetrabenazine treatment was generally efficacious, safe, and well tolerated in patients with TD.

Presented at: The American Psychiatric Association 2017 Annual Meeting; May 20-24, 2017; San Diego, California, USA.

FUNDING ACKNOWLEDGEMENTS: This study was funded by Teva Pharmaceutical Industries, Petach Tikva, Israel.

150

\section{Estimation of an MCID for AIMS Total Score Change in Tardive Dyskinesia}

Martha Sajatovic, $\mathrm{MD}^{\prime}$; Andrew J. Cutler, $\mathrm{MD}^{2}$; Khodayar Farahmand, PharmD ${ }^{3}$; Joshua Burke, $M S^{3}$; Scott Siegert, PharmD ${ }^{4}$; and Grace S. Liang, $M D^{5}$

${ }^{1}$ Neurological and Behavioral Outcomes Center, University Hospitals Cleveland Medical Center, Cleveland, $\mathrm{OH}$

${ }^{2}$ Meridien Research, Tampa, FL

${ }^{3}$ Director, Head of Medical Communications, Neurocrine Biosciences, Inc., San Diego, CA

${ }^{4}$ Executive Director, Head of Medical Affairs, Neurocrine Biosciences, Inc., San Diego, CA

${ }^{5}$ Medical Director, Clinical, Neurocrine Biosciences, Inc., San Diego, CA

ABSTRACT: Background: The efficacy of valbenazine (INGREZZA) in tardive dyskinesia (TD) was demonstrated in placebo-controlled clinical trials, based on the Abnormal Involuntary Movement Scale (AIMS) total score (sum of items 1-7). In these trials, mean changes in the AIMS total score were significantly greater with valbenazine $80 \mathrm{mg}$ than with placebo. Currently, no minimal clinically important difference (MCID) has been established for the AIMS total score in patients with TD. Using valbenazine trial data, analyses were conducted to establish a MCID for AIMS total score in TD.

METHODS: Data were pooled from three 6-week trials: KINECT (NCT01688037), KINECT 2 (NCT01733121), KINECT 3 (NCT02274558). Using the Clinical Global
Impression ofChange (CGI-TD) as an anchor comparison, AIMS total score changes from baseline to Week 6 were summarized for all study participants (pooled valbenazine and placebo groups) with a "minimal" CGITD score of $\leq 3$ (minimally improved or better) or "robust" $\leq 2$ (much improved or better) at Week 6 .

RESULTS: In the pooled population ( $\mathrm{N}=373), 72 \%$ and $29 \%$ of all participants had CGI-TD scores of $\leq 3$ and $\leq 2$, respectively. The median (maximum, minimum) change from baseline in AIMS total score at Week 6 was -2 $(-13,8)$ in participants with CGI-TD score $\leq 3$ and -3 $(13,8)$ in participants with a score $\leq 2$.

CONCLUSION: Pooled data from 3 randomized, doubleblind, placebo-controlled trials suggest that a 2 point decrease in AIMS total score may represent the minimal clinically meaningful improvement. Larger AIMS score improvements were associated with "much improved" or "very much improved" CGI TD assessments.

FUNDING ACKNOWLEDGEMENTS: This study was funded by Neurocrine Biosciences, Inc.

\section{1}

\section{Improving the Systematic Use of Pharmacogenetic Testing for Depression Prescribing \\ Lauren Thomann Hughes, DNP, ARNP, PMHNP-BC}

Psychiatric/Mental Health Nurse Practitioner, Abbe Center for Community Mental Health, Iowa City, IA

ABSTRACT: Study Objectives: The purpose of this project was to systematize the use of pharmacogenetic testing (PGT) among psychiatric prescribers. The use of PGT in clinical practice is inconsistent despite the evidence supporting its efficacy (Burke, Love, Jones, \& Fife, 2016). The question to be answered is: In patients with major depressive disorder (MDD), how is PGT currently used in clinical practice compared to use after implementation of practice change interventions?

METHOD: This study was conducted among 4 psychiatric prescribers in a behavioral health clinic. 3 interventions were utilized to change practice. An educational inservice was delivered to address the PGT knowledge gap. A protocol for identifying patients that may benefit from PGT was developed, indicating PGT was warranted for patients with non-remitting moderate to severe MDD and at least 2 medication failures from 2 different classes. Next, a medication failure documentation template and the PGT report were integrated into the EHR. A baseline survey was administered before the in-service, assessing 